\title{
Relevância do grau de incapacidade física como preditor do diagnóstico tardio em
}

\section{hanseníase: Uma revisão de escopo}

\author{
Relevance of the degree of physical disability as a predictor of late diagnosis in leprosy: A scoping \\ review
}

Relevancia del grado de discapacidad física como predictor del diagnóstico tardío en la lepra: Una revisión del alcance

\author{
Gracinda Maria Gomes Alves \\ ORCID: https://orcid.org/0000-0002-7556-1123 \\ Universidade Federal de Sergipe, Brasil \\ E-mail: gracindamga@gmail.com \\ Alda Graciele Claudio dos Santos Almeida \\ ORCID: https://orcid.org/ 0000-0003-0406-8849 \\ Universidade Federal de Alagoas, Brasil \\ Curso de Medicina do CESMAC, Brasil \\ E-mail: alda.almeida@eenf.ufal.br \\ Mirana Moura Licetti \\ ORCID: https://orcid.org/0000-0003-4787-5683 \\ Universidade Federal de Alagoas, Brasil \\ E-mail: licettimirana@gmail.com \\ Caroline Magna de Oliveira Costa \\ ORCID: https://orcid.org/0000-0003-4096-4093 \\ Universidade Federal de Alagoas, Brasil \\ E-mail: carolmagnacosta@gmail.com \\ Karina Conceição Gomes Machado de Araújo \\ ORCID: https://orcid.org/0000-0003-4433-5227 \\ Universidade Federal de Sergipe, Brasil \\ E-mail: karinaconceicaoaraujo@gmail.com
}

\begin{abstract}
Resumo
O grau de incapacidade física é um indicador que pode determinar a presença de diagnóstico tardio e ineficácia nas ações de controle. O objetivo desta revisão foi analisar os artigos que apresentaram evidências científicas sobre a avaliação do grau de incapacidade física como preditor de diagnóstico tardio em hanseníase. A metodologia utilizada seguiu a estratégia de busca recomendada pelo The Joanna Briggs Institute Reviewers' Manual, assim como o protocolo PRESS (Peer Review of Electronic Search Strategies), a pesquisa foi realizada em 3 etapas, usando as palavras-chave e executada por dois pesquisadores de forma independente, nas seguintes bases de dados: Scopus, National Library of Medicines (NLM) - MEDLINE via Pubmed, Web Of Science, Cochrane Library e Biblioteca Virtual em Saúde (BVS). Dos 510 artigos, inicialmente, selecionados resultou numa eleição de sete artigos analisados. Nos resultados foi observada uma frequência expressiva de casos novos com registro do grau de incapacidade física e com a classificação operacional multibacilar, indicadores estes que confirmam o atraso no diagnóstico. A conclusão deste estudo evidencia que o grau de incapacidade física é relevante no diagnóstico da hanseníase como indicador e preditor do diagnóstico tardio e possivelmente de endemia oculta.
\end{abstract}

Palavras-chave: Pessoas com deficiência; Hanseníase; Diagnóstico tardio.

\begin{abstract}
The degree of physical disability is an indicator that can determine the presence of late diagnosis and ineffective control actions. The objective of this review was to analyse the articles that presented scientific evidence on the evaluation of the degree of physical disability as a predictor of late diagnosis in leprosy. The methodology used followed the search strategy recommended by The Joanna Briggs Institute Reviewers' Manual, as well as the PRESS protocol (Peer Review of Electronic Search Strategies), the search was carried out in 3 stages, using the keywords and performed by two authors independently, in databases: Scopus, National Library of Medicines (NLM) - MEDLINE via Pubmed, Web Of Science, Cochrane Library e Biblioteca Virtual em Saúde (BVS). Of the 510 articles initially selected, an election of seven analyzed articles resulted. In the results it was observed an expressive frequency of new cases with a record of the degree of physical disability and with the multibacillary operational classification, indicators that corroborate the delay in diagnosis. The conclusion of this study shows that the degree of physical
\end{abstract}


disability is relevant in the diagnosis of leprosy as an indicator and predictor of late diagnosis and possibly of hidden endemicity.

Keywords: Disabled persons; Leprosy; Delayed diagnosis.

\begin{abstract}
Resumen
El grado de discapacidad física es un indicador que puede determinar la presencia de un diagnóstico tardío y de acciones de control ineficaces. El objetivo de esta revisión fue analizar los artículos que presentaban evidencia científica sobre la evaluación del grado de discapacidad física como predictor del diagnóstico tardío en la lepra. La metodología utilizada siguió la estrategia de búsqueda recomendada por el Manual de Revisores del Instituto Joanna Briggs, así como el protocolo PRESS (Peer Review of Electronic Search Strategies), la búsqueda se realizó en 3 etapas, utilizando las palabras clave y realizada por dos autores de forma independiente, en las bases de datos: Scopus, National Library of Medicines (NLM) - MEDLINE via Pubmed, Web Of Science, Cochrane Library e Biblioteca Virtual em Saúde (BVS). De los 510 artículos seleccionados inicialmente se eligieron siete artículos analizados. En los resultados se observó una frecuencia expresiva de nuevos casos con registro del grado de incapacidad física y con la clasificación operacional multibacilar, indicadores que probar un retraso en el diagnóstico. La conclusión de este estudio muestra que el grado de discapacidad física es relevante en el diagnóstico de la lepra como indicador y predictor de diagnóstico tardío y posiblemente de endemicidad oculta.
\end{abstract}

Palabras clave: Personas con discapacidad; Lepra; Diagnóstico tardio.

\title{
1. Introdução
}

A Hanseníase é uma doença dermatoneurológica, crônica, infectocontagiosa, de evolução lenta que tem como agente etiológico o Micobacterium Leprae, definido como um bacilo que sobrevive no meio intracelular e que afeta preferencialmente a pele, os nervos e a mucosa. A transmissão ocorre através de gotículas eliminas do aparelho respiratório superior de pessoas acometidas pelo bacilo, que apresentam carga bacilar elevada e o diagnóstico é dado através da avaliação clínica que inclui o exame dermatoneurológico. Durante sua evolução, a hanseníase é uma doença que pode ocasionar episódios reacionais com danos graves aos troncos nervosos periféricos resultando em incapacidades físicas (Talhari, Penna, Gonçalves \& Oliveira, 2015).

Em busca de soluções a Organização Pan-Americana da Saúde em acordo com a Organização Mundial da Saúde (OPAS/OMS) apresentou a Estratégia Global para Hanseníase 2016-2020 baseada no combate à hanseníase e suas complicações propondo parcerias que viessem também a fortalecer a inclusão e extinguir a discriminação. Outros países também enfrentam a doença como um problema de saúde pública e nas Américas o Brasil se situa neste panorama de alta prevalência na detecção de casos novos (OPAS/OMS, 2017).

Atualmente o Brasil é o segundo país na lista de detecção de casos novos por 100 mil habitantes na população em geral. Em uma série histórica dos anos de 2009 -2018 apresentou oscilações para menos no parâmetro de endemicidade e da presença do grau de incapacidade física no diagnóstico que começou com 12,70/ 1 milhão de habitantes (GIF2) e no último ano da série mostra um registro de casos novos com a taxa de 10,08/ 1 milhão de habitantes com GIF 2 no diagnóstico inicial. Todavia o estado do Tocantins apresentou uma taxa deste indicador excessivamente alta (84,87/1 milhão de habitantes). (Brasil. Boletim Epidemiológico, 2020).

A taxa do grau incapacidade física nas pessoas afetadas pela hanseníase encontrada nos registros é um indicador que reflete os procedimentos e efetividade das ações de controle como também aponta as condições de acesso desta população aos serviços de atendimento. O GIF pode ser classificado em: GIF0, sem comprometimento nos segmentos avaliados dos olhos, pés e mãos; GIF1, quando é observada alteração neural nos segmentos avaliados, como diminuição ou perda de sensibilidade ou da força muscular em olhos, pés e mãos; e GIF2, quando apresenta na avaliação dos segmentos examinados deformidades, como garras nas mãos ou nos pés, reabsorção óssea, mão ou pé caído, atrofia muscular e alterações oculares como lagoftalmo, iridociclite, opacidade corneana e perda da acuidade visual. Este indicador mostra a realidade das ações para a prevenção das incapacidades bem como sinaliza o diagnóstico tardio (Brasil, 2016). Diante desse contexto, o objetivo desta revisão foi 
analisar os artigos que apresentaram evidências científicas sobre a avaliação do grau de incapacidade física como preditor de diagnóstico tardio em hanseníase.

\section{Metodologia}

\section{Tipo de estudo}

Trata-se de um estudo metodológico do tipo revisão de escopo ("Scoping Review"), de natureza quantitativa, que compreende um tipo de revisão de literatura, possuindo como técnica mapear os principais conceitos, clarificar áreas de pesquisa, orientar para futuras evidências e identificar lacunas do conhecimento.

O presente estudo seguiu rigorosamente as recomendações estabelecidas no manual de revisão de escopo do Joanna Briggs Institute (JBI), versão 2020 (Peters et al., 2020), assim como no protocolo PRESS (Peer Review of Electronic Search Strategies).

\section{Identificação da pergunta de pesquisa}

Para a construção da pergunta da pesquisa, utilizou-se a estratégia mnemônica PCC para a scoping review, em que P Population (População), C- Concept (Conceito) e C - Context (Contexto) (Peters et al., 2020). Foram definidos: P- pacientes com hanseníase; C- avaliação do grau de incapacidade física como preditor de diagnóstico tardio e C: sem contexto determinado. Com base nessas definições foi estabelecida a pergunta norteadora: "Quais são as evidências científicas sobre a avaliação do grau de incapacidade física como preditor de diagnóstico tardio em hanseníase?”

\section{Estratégia da pesquisa}

A estratégia de busca teve como objetivo encontrar estudos publicados em periódicos indexados. Foram definidos os descritores e palavras-chaves conforme os Descritores em Ciências da Saúde (DeCS) e Medical Subject Headings (MeSH) e para a combinação dos mesmos foi utilizado os operadores booleanos ( $A N D, O R$ e NOT) formando os cruzamentos para busca conforme as especificidades das bases de dados. A busca foi realizada por dois pesquisadores independentes, conforme critérios estabelecidos pelo JBI (Peters et al, 2020).

A estratégia de busca foi conduzida em três etapas: I) A busca foi realizada em duas bases de dados: Medical Literature Analysis and Retrieval System Online (MEDLINE) e SCOPUS, para validação da estratégia de busca e dos cruzamentos, analisando títulos e resumos dos artigos identificados que atendessem aos critérios de inclusão. Desse modo, após a validação da estratégia de busca e dos cruzamentos, foi definido que a estratégia de pesquisa utilizada seria com os seguintes descritores articulados com os operadores booleanos: Leprosy AND Physical disability OR Disabled Persons AND Delayed Diagnosis. II) A pesquisa efetuou-se utilizando palavras-chave e descritores, nas seguintes bases de dados e portais: Scopus, National Library of Medicines (NLM) - MEDLINE via Pubmed, Web Of Science, Cochrane Library e Biblioteca Virtual em Saúde (BVS). III) Executou-se uma pesquisa nas listas de referência de todos os estudos incluídos na revisão.

\section{Seleção dos estudos}

Para a seleção dos estudos efetuou-se a leitura criteriosa do título, resumo e descritores/palavras-chave, e, posteriormente, leitura na íntegra dos estudos em consonância com os critérios de elegibilidade estabelecidos. Critérios de inclusão: a) estudos que atendessem aos objetivos propostos respondendo à estratégia: População, Conceito, Contexto (PCC); b) tipos de fontes de evidência: pesquisas primárias, revisões sistemáticas, metanálises e diretrizes/guidelines; c) estudos realizados nos idiomas inglês, espanhol e português; d) estudos com acesso aberto do tipo open access e com acesso pelo portal de periódicos CAPES via Instituição de Ensino Superior; e) não houve restrição quanto ao ano de publicação. Critérios 
de exclusão: Para esta revisão foram excluídos os estudos que não estão publicados na íntegra, publicações de opiniões, consensos, retrações, editoriais e websites.

Neste sentido, o levantamento bibliográfico foi realizado no período de setembro a dezembro de 2020, a seleção dos estudos foi realizada por dois revisores e na presença de qualquer divergência foi consultado um terceiro revisor. Foi utilizado o fluxograma do Statement for Reporting Systematic Reviews and Meta-Analyses of Studie - PRISMA detalhando o fluxo da pesquisa, através da seleção da fonte, duplicatas, recuperação de texto completo e quaisquer adições da terceira pesquisa, dados de extração e apresentação da evidência. E para o gerenciamento de referências utilizou-se o software EndNote, principalmente, para seleção e exclusão dos estudos duplicados.

\section{Mapear os dados}

$\mathrm{Na}$ etapa de mapeamento dos dados, utilizou-se um instrumento estruturado conforme recomendações do JBI, com as seguintes informações: autor, ano de publicação, país de origem, objetivo do estudo, população do estudo, método do estudo, resultados: grau de incapacidade física ao diagnóstico, outros detalhes e achados que relatam as questões pertinentes à revisão de escopo.

\section{Análise das evidências}

Posteriormente, na etapa de análise das evidências, realizou-se um mapeamento descritivo dos estudos selecionados.

\section{Agrupar, resumir e apresentar os resultados}

Ao dar seguimento à pesquisa com essa etapa, os dados dos estudos foram agrupados por eixos temáticos, sendo os resultados apresentados utilizando-se de tabelas e resumo descritivo

\section{Resultados}

Na etapa de identificação, foram encontrados 510 artigos nas bases de dados e após análise das referências, 16 artigos foram adicionados. Posteriormente, na etapa de seleção foram eliminados 41 artigos duplicados, dessa maneira, totalizando 485 artigos selecionados, dos quais após a análise de títulos e resumos foram excluídos 435 artigos.

Em seguida, na etapa de elegibilidade, 50 artigos completos foram avaliados, após a leitura na íntegra destes, foram excluídos 43 artigos, por não atenderem à estratégia PCC ou por serem artigos de opinião. Nesta revisão, a amostra final totalizou em 7 artigos selecionados. O processo de busca e seleção dos estudos desta revisão está apresentado no fluxograma (Figura 1), conforme recomendações do JBI, segundo checklist adaptado do Preferred Reporting Items for Systematic Reviews and Meta-Analyses (PRISMA). 
Figura 1. Fluxograma do processo de seleção dos estudos, adaptado do PRISMA. Maceió, AL, Brasil, 2021.

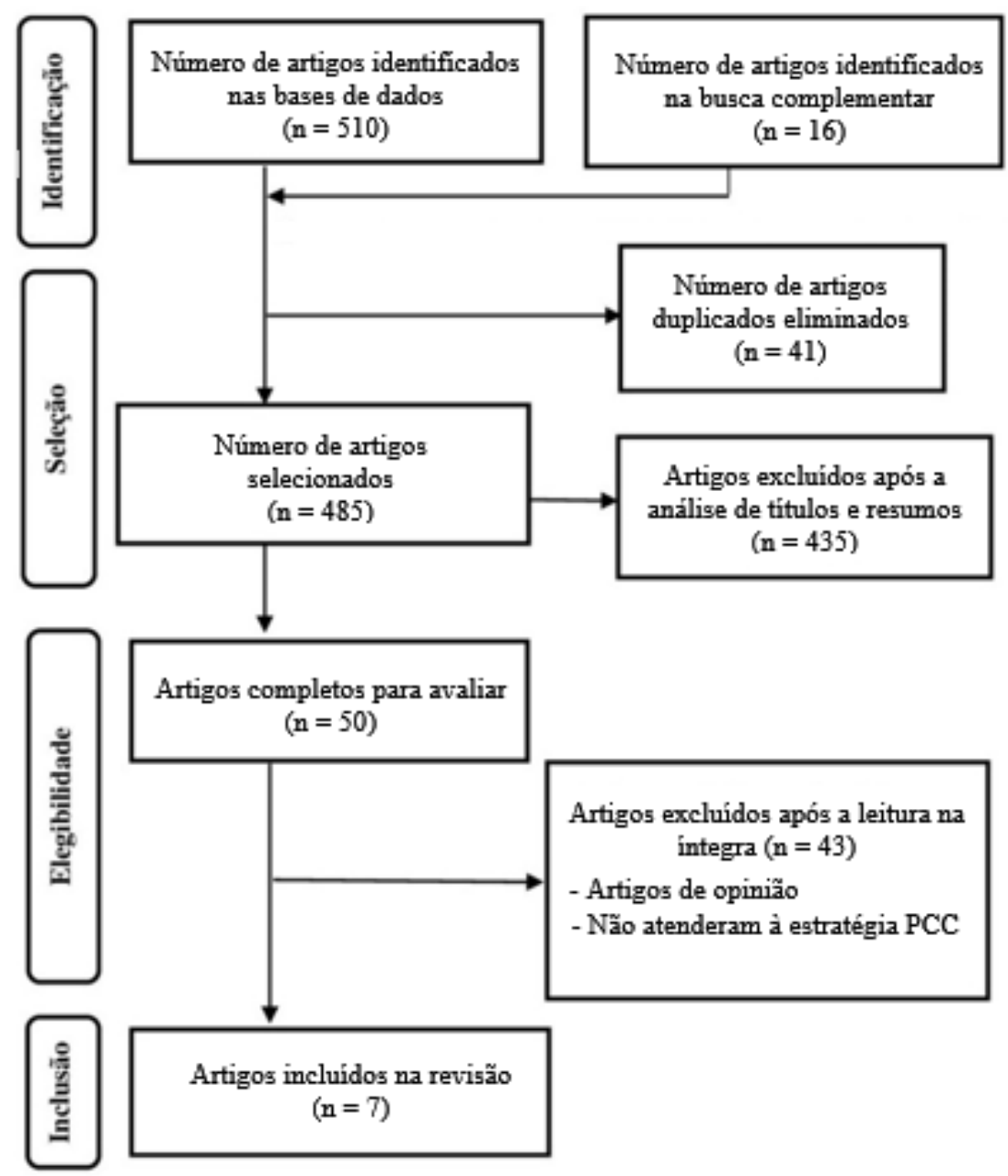

Fonte: Autores.

No Quadro 1 apresenta a seleção dos estudos que atenderam aos critérios estabelecidos. Neste quadro observa-se dados e descrições relativas aos estudos selecionados em que nos resultados e nas conclusões os autores identificaram a presença da incapacidade física e destacaram a possibilidade de um diagnóstico ter sido tardio, bem como a suspeição de endemia oculta. 
Quadro 1. Relação de estudos selecionados: título, autor, ano de publicação, país de origem, objetivo do estudo, metodologia, resultados e conclusão. Maceió, AL, Brasil, 2021.

\begin{tabular}{|c|c|c|c|c|c|c|c|}
\hline Estudo & Título & Autor & $\begin{array}{c}\text { País de } \\
\text { origem e } \\
\text { ano de } \\
\text { publicação }\end{array}$ & $\begin{array}{c}\text { Objetivo do } \\
\text { estudo }\end{array}$ & Método & Resultados & Conclusão \\
\hline E1 & $\begin{array}{l}\text { Clinical and } \\
\text { epidemiological } \\
\text { profile of leprosy } \\
\text { patients attended at } \\
\text { Ceará, 2007-2011 }\end{array}$ & $\begin{array}{l}\text { Queirós, M.I., } \\
\text { Ramos, J.A.N., } \\
\text { Alencar, } \\
\text { C.H.M., } \\
\text { Monteiro, } \\
\text { L.D., Sena, } \\
\text { A.L. \& } \\
\text { Barbosa, J. C. }\end{array}$ & $\begin{array}{l}\text { Brasil, } \\
2016\end{array}$ & $\begin{array}{l}\text { Caracterizar o } \\
\text { perfil clínico } \\
\text { epidemiológico da } \\
\text { população } \\
\text { estudada. }\end{array}$ & $\begin{array}{l}\text { Descritivo } \\
\text { de } \\
\text { natureza } \\
\text { retrospecti } \\
\text { va. }\end{array}$ & $\begin{array}{l}\text { Do total de casos } \\
\text { novos avaliados, } \\
20,5 \% \\
\text { apresentavam } \\
\text { incapacidades } \\
\text { físicas. }\end{array}$ & $\begin{array}{l}\text { O estudo revela } \\
\text { endemia oculta e } \\
\text { atraso no } \\
\text { diagnóstico. }\end{array}$ \\
\hline E2 & $\begin{array}{l}\text { Retraso en el } \\
\text { diagnóstico de lepra } \\
\text { como factor } \\
\text { pronóstico de } \\
\text { discapacidad en una } \\
\text { cohorte de pacientes } \\
\text { en Colombia, 2000- } \\
2010\end{array}$ & $\begin{array}{l}\text { Guerrero, M. } \\
\text { I., Muvdi, S. \& } \\
\text { León, C. I. }\end{array}$ & $\begin{array}{l}\text { Colômbia, } \\
2013\end{array}$ & $\begin{array}{l}\text { Avaliar fatores } \\
\text { prognósticos para } \\
\text { incapacidade no } \\
\text { diagnóstico inicial. }\end{array}$ & $\begin{array}{l}\text { Estudo de } \\
\text { coorte } \\
\text { retrospecti } \\
\text { va. }\end{array}$ & $\begin{array}{l}\text { Os resultados } \\
\text { confirmam que há } \\
\text { prevalência oculta } \\
\text { na área do estudo } \\
\text { considerando a } \\
\text { importância da } \\
\text { avaliação do GIF. }\end{array}$ & $\begin{array}{l}\text { A presença da } \\
\text { incapacidade } \\
\text { física depende do } \\
\text { tempo de atraso } \\
\text { no diagnóstico. }\end{array}$ \\
\hline E3 & $\begin{array}{l}\text { Factors associated } \\
\text { with the delay of } \\
\text { diagnosis of leprosy } \\
\text { in north-eastern } \\
\text { Colombia: a } \\
\text { quantitative analysis }\end{array}$ & $\begin{array}{l}\text { Gómez, L., } \\
\text { Rivera, A., } \\
\text { Vidal ,Y., } \\
\text { Bilbao, J., } \\
\text { Kasang C. \& } \\
\text { Parisi, S. et al. }\end{array}$ & $\begin{array}{l}\text { Colômbia, } \\
2018\end{array}$ & $\begin{array}{l}\text { Determinar os } \\
\text { fatores associados } \\
\text { ao atraso no } \\
\text { diagnóstico. }\end{array}$ & $\begin{array}{c}\text { Estudo } \\
\text { observacio } \\
\text { nal. }\end{array}$ & $\begin{array}{l}\text { No momento do } \\
\text { diagnóstico, } 14,9 \% \\
\text { dos } 249 \text { casos } \\
\text { novos } \\
\text { apresentavam GIF } \\
\text { II. }\end{array}$ & $\begin{array}{lr}\text { A taxa alta de } \\
\text { GIF2 no } \\
\text { momento do } \\
\text { diagnóstico de } \\
\text { casos novos pode } \\
\text { ser explicada } \\
\text { pelo atraso no } \\
\text { diagnóstico. }\end{array}$ \\
\hline E4 & $\begin{array}{l}\text { Clinical variables } \\
\text { associated with } \\
\text { leprosy reactions and } \\
\text { persistence of } \\
\text { physical impairment }\end{array}$ & $\begin{array}{l}\text { Oliveira, } \\
\text { D.T.D., } \\
\text { Sherlock, J., } \\
\text { Melo, E.V.D., } \\
\text { Rollemberg, } \\
\text { K.C.V., } \\
\text { Paixao, } \\
\text { T.R.S.D., } \\
\text { Abuawad, } \\
\text { Y.G. et al }\end{array}$ & $\begin{array}{l}\text { Brasil, } \\
2013\end{array}$ & $\begin{array}{l}\text { Avaliar os fatores } \\
\text { clínicos associados } \\
\text { à ocorrência de } \\
\text { reações hansênicas } \\
\text { e grau de } \\
\text { incapacidade. }\end{array}$ & $\begin{array}{c}\text { Estudo } \\
\text { retrospecti } \\
\text { vo. }\end{array}$ & $\begin{array}{l}\text { A maioria dos } \\
\text { casos novos era de } \\
\text { multibacilares e } \\
\text { muitos com GIF2. }\end{array}$ & $\begin{array}{l}\text { Desenvolver } \\
\text { estratégias para } \\
\text { realização do } \\
\text { exame } \\
\text { dermatoneurológ } \\
\text { ico antes e } \\
\text { depois do } \\
\text { tratamento. }\end{array}$ \\
\hline E5 & $\begin{array}{l}\text { Evaluation of the } \\
\text { degree of incapacity } \\
\text { of patients with a } \\
\text { diagnosis of leprosy } \\
\text { at a dermatology } \\
\text { service in the State of } \\
\text { São Paulo }\end{array}$ & $\begin{array}{l}\text { Alves, C. J. } \\
\text { M., Barreto, J. } \\
\text { A., Fogagnolo, } \\
\text { L., Contin, L. } \\
\text { A. \& Nassif, P. } \\
\text { W. }\end{array}$ & $\begin{array}{l}\text { Brasil, } \\
2010\end{array}$ & $\begin{array}{l}\text { Investigar o } \\
\text { registro do grau de } \\
\text { incapacidade física } \\
\text { no momento do } \\
\text { diagnóstico. }\end{array}$ & $\begin{array}{c}\text { Estudo } \\
\text { transversal } \\
\text { observacio } \\
\text { nal. }\end{array}$ & $\begin{array}{l}\text { Relação direta } \\
\text { entre o GIF e o } \\
\text { tempo de duração } \\
\text { da doença. }\end{array}$ & $\begin{array}{l}\text { O diagnóstico } \\
\text { tardio leva à } \\
\text { sustentação da } \\
\text { endemia. }\end{array}$ \\
\hline E6 & $\begin{array}{l}\text { Clinical variables } \\
\text { associated to the } \\
\text { degree of physical } \\
\text { disability in leprosy }\end{array}$ & $\begin{array}{l}\text { Silva J.S.R, } \\
\text { Palmeira I.P., } \\
\text { Sá A.M.M., } \\
\text { Nogueira } \\
\text { L.M.V., } \\
\text { Ferreira } \\
\text { A.M.R. }\end{array}$ & $\begin{array}{l}\text { Brasil, } \\
2019\end{array}$ & $\begin{array}{l}\text { Analisar a } \\
\text { associação entre as } \\
\text { vaiáveis clínicas e } \\
\text { os graus de } \\
\text { incapacidade física } \\
\text { em casos novos de } \\
\text { hanseníase. }\end{array}$ & $\begin{array}{c}\text { Estudo } \\
\text { transversal } \\
\text { e analítico }\end{array}$ & $\begin{array}{l}\text { O maior percentual } \\
\text { total de casos } \\
\text { novos com GIF } \\
\text { associado ao maior } \\
\text { percentual de casos } \\
\text { novos } \\
\text { multibacilares. }\end{array}$ & $\begin{array}{l}\text { Mais efetividade } \\
\text { em prevenção de } \\
\text { incapacidade e } \\
\text { diagnóstico } \\
\text { precoce. }\end{array}$ \\
\hline
\end{tabular}




\begin{tabular}{|c|c|c|c|c|c|c|c|}
\hline E7 & $\begin{array}{l}\text { Disability among } \\
\text { new leprosy patients } \\
\text { an issue of concem: } \\
\text { An institution based } \\
\text { study in an endemic } \\
\text { district for leprosy, in } \\
\text { the state of West } \\
\text { Bengal, India. }\end{array}$ & $\begin{array}{l}\text { Silva, J.S.R, } \\
\text { Palmeira, I. P; } \\
\text { Sá, A.M.M; } \\
\text { Nogueira, } \\
\text { L.M.V \& } \\
\text { Ferreira, } \\
\text { A.M.R. }\end{array}$ & $\begin{array}{l}\text { Índia, } \\
2012\end{array}$ & $\begin{array}{lr}\text { Descobrir } & \text { a } \\
\text { prevalência do grau } \\
\text { de incapacidade } \\
\text { física nos } & \text { casos } \\
\text { novos } & \text { na } \\
\text { população } & \\
\text { estudada. } & \end{array}$ & $\begin{array}{l}\text { Estudo } \\
\text { transversal }\end{array}$ & $\begin{array}{l}\text { Percentual elevado } \\
\text { dos casos novos } \\
\text { apresentou } \\
\text { incapacidades } \\
\text { físicas no momento } \\
\text { do diagnóstico. }\end{array}$ & $\begin{array}{l}\text { Exame } \\
\text { dermatoneurológ } \\
\text { ico } \\
\text { no momento do } \\
\text { diagnóstico } \\
\text { precisa ser } \\
\text { obrigatório. }\end{array}$ \\
\hline
\end{tabular}

Legenda: Os artigos estão codificados numericamente (E1, E2, E3, E4, E5, E6 e E7), citados desta forma. Fonte: Autores.

O estudo (E1) de Queiroz et al (2016) no Estado do Ceará, Brasil, empregou a metodologia retrospectiva e descreveu nos resultados que nos casos novos em que o GIF foi avaliado no momento do diagnóstico, um total de $20,5 \%$ apresentou incapacidades físicas, destes 57,4\% foram classificados com o GIF 1 e 42,6\% foram classificados com o GIF2. No entanto, no período do estudo (2007 a 2011) foram diagnosticados e tratados no referido serviço 475 casos novos, dos quais 458 casos novos foram avaliados quanto ao GIF. Isto significa que 17 pacientes $(3,5 \%)$ não fizeram avaliação do grau de incapacidade no diagnóstico inicial. O estudo mostra também que os casos novos com incapacidade física apresentaram uma maior proporção $(31,1 \%)$ nos pacientes com a classificação operacional MB.

Um estudo analítico de coorte retrospectivo (E2) desenvolvido na Colômbia por Guerrero et al (2013) buscou avaliar fatores prognósticos para a presença de incapacidades físicas no momento do diagnóstico. No intervalo deste estudo (2000 a 2010) $9,8 \%$ dos casos novos diagnosticados apresentaram incapacidades físicas como também foi notada uma maior frequência de incapacidades físicas em paciente MB.

Em outra pesquisa desenvolvida por L. Goméz et al (2018) em um município endêmico na Colômbia (E3), 249 pacientes foram diagnosticados no período do estudo (2011 a 2015), destes 63\% eram pacientes multibacilares e 39,8\% do total apresentavam alguma incapacidade física no momento do diagnóstico.

Na pesquisa realizada em um município do Nordeste do Brasil (Aracajú/Sergipe) (E4) foi feito um levantamento dos dados de registro nos prontuários entre os anos de 2005 até 2011 e identificaram que dos 494 pacientes inscritos para tratamento neste referido serviço, tiveram um percentual de diagnósticos da forma MB e PB similares. No entanto, 396 $(80,2 \%)$ foram avaliados quanto ao grau de incapacidade física no momento do diagnóstico, destes 36\% apresentavam incapacidades físicas entre grau 1 e 2 (Oliveira et al, 2013).

Alves et al (2010) realizaram uma investigação (E5) nos prontuários de 167 pacientes de um serviço de atendimento ao paciente de hanseníase no município de São Paulo/Brasil no período de 2003 a 2007, em busca do registro da avaliação do GIF no momento do diagnóstico. Nos resultados observaram que $60 \%$ apresentavam alterações na capacidade física já no diagnóstico inicial e destes 34\% estavam com o registro de GIF1 e 26\% com GIF2. Nesta amostra a classificação operacional MB no diagnóstico da hanseníase foi registrada em $73 \%$ dos pacientes.

Pesquisadores realizaram um estudo (E6) transversal e analítico no estado do Pará em um centro de referência no atendimento ao paciente com hanseníase e encontraram nos resultados uma predominância da forma $\mathrm{MB}$ em $77,1 \%$ dos 249 prontuários analisados, deste 17,7\% estavam com GIF 1 e 10,5\% com GIF2 (Silva et al, 2019).

No estudo transversal (E7) desenvolvido em Bengala na Índia os pesquisadores avaliaram 244 casos novos entre agosto de 2006 e junho de 2007 e os resultados mostraram que 20,1\% apresentavam incapacidades físicas, o GIF 1 foi registrado em $11,5 \%$ destes pacientes e o GIF 2 em 8,6\% (Sarkar et al, 2012).

A Tabela 1 apresenta o número total da amostra e o número de pacientes em que o grau de incapacidade física foi avaliado no momento do diagnóstico. Desta forma podem ser observados índices significativos do GIF 1 e GIF 2 no diagnóstico dos casos novos das pessoas afetadas pela hanseníase como também uma percentagem importante de casos 
multibacilares. Destes estudos, dois mostram dados com discrepância entre o total de casos novos registrados e o número de casos novos registrados com avaliação neurológica, isto é, do grau de incapacidade física no momento da avaliação inicial.

Tabela 1. Número do total de casos novos das pessoas afetadas pela hanseníase registrado nas amostras dos estudos citados, número total e porcentagem dos pacientes que foram avaliados quanto ao grau de incapacidade física 1 e 2 no momento do diagnóstico e a classificação operacional registrada. Maceió, AL, Brasil, 2021.

\begin{tabular}{ccccccc}
\hline ESTUDO & $\begin{array}{c}\text { Número total da } \\
\text { amostra (casos } \\
\text { novos) }\end{array}$ & $\begin{array}{c}\text { Número de casos novos } \\
\text { avaliados quanto ao GIF no } \\
\text { diagnóstico }\end{array}$ & GIF 1 (\%) & GIF 2(\%) & MB \% & PB \% \\
\hline E1 & 475 & 458 & $57,4 \%$ & $42,6 \%$ & $65,4 \%$ & $34,6 \%$ \\
E2 & 333 & 333 & $19,8 \%$ & $15,3 \%$ & $67,9 \%$ & $32,1 \%$ \\
E3 & 249 & 249 & $14,9 \%$ & $24,9 \%$ & $63 \%$ & $37 \%$ \\
E4 & 494 & 396 & $27 \%$ & $9 \%$ & $49,2 \%$ & $50,8 \%$ \\
E5 & 167 & 167 & $34 \%$ & $26 \%$ & $73 \%$ & $27 \%$ \\
E6 & 323 & 323 & $17,7 \%$ & $10,5 \%$ & $77,1 \%$ & $22,9 \%$ \\
E7 & 244 & 244 & $11,5 \%$ & $8,6 \%$ & $46,7 \%$ & $53,2 \%$ \\
\hline
\end{tabular}

Legenda: GIF (grau de incapacidade física). Classificação operacional: MB (multibacilar), PB (paucibacilar). Fonte: Autores.

\section{Discussão}

A WHO (2020) na recém lançada companha da Global Leprosy Estrategy, 2021-2030, em busca da eliminação da doença, da incapacidade física e da discriminação e estigma aponta como um dos desafios atuais o atraso no diagnóstico. É certo que a presença de deformidades visíveis compatíveis com as lesões de nervos periféricos encontradas na hanseníase caracteriza o diagnóstico. No manual de monitoramento, lançado pela World Health Organization (2017), o registro do GIF 2 é apontado como um indicador que deve ser aferido nos casos novos para a elaboração das metas a serem alcançadas pelas estratégias de controle e eliminação da hanseníase e tem importância para o acompanhamento e evolução da doença. Este indicador em menores de 15 anos é determinante em relação à situação de controle da hanseníase. Portanto, a relevância desta informação é significativa para a compreensão da amplitude e intensidade da endemia (WHO: 2016, 2017; Barros et al, 2016).

De acordo com o Manual técnico-operacional, do Ministério da Saúde (Brasil, 2016), a avaliação da função neural e o grau de incapacidade física no momento do diagnóstico é indispensável e está contemplada entre as medidas que visam prevenir incapacidades físicas, evitar problemas socioeconômicos decorrentes como também dificuldades emocionais (Brasil. MS, 2016).

A falta de normas e registros acurados como também de capacitação das equipes nos serviços de saúde que prestam assistência às pessoas diagnosticadas com hanseníase podem levar às subnotificações como também ao erro nas avaliações e nos prontuários. O levantamento e registro da proporção do indicador GIF, é importante principalmente quando associado à proporção dos casos multibacilares, e tem importância singular quando avaliado no momento do diagnóstico (WHO, 2017). De tal modo que um erro na notificação dos dados da classificação operacional ou uma subnotificação dos dados do GIF podem afetar a produção e desenvolvimento dos programas e ações de controle e eliminação da hanseníase como um grave problema de saúde pública. Observa-se nos artigos, analisados por esta revisão, que a avaliação do GIF foi realizada, todavia em alguns artigos não foi observada uma avaliação do GIF no total da amostra.

Na pesquisa do artigo E1 (Queirós et al, 2016) os autores fizeram uma ressalva quanto às possíveis limitações do estudo decorrente dos registros no serviço referido mesmo tendo usado também fontes como o Serviço de Informação e 
Notificação de Agravos (SINAN) objetivando aprimorar os resultados. Pontuam a necessidade de monitoramento e supervisão das unidades de referência, pois estes autores entenderam que a incapacidade física deve ser diagnosticada precocemente. Acrescentam que a presença de incapacidade no momento do diagnóstico acena para uma possível endemia oculta, isto é, casos que não estão sendo diagnosticados.

Outra observação, descrita no artigo E1(Queirós et al, 2016), revela uma divergência na forma de classificação em relação ao preconizado pela OMS. Na classificação operacional recomendada pela OMS (WHO, 2017), o diagnóstico da doença é correspondente ao número de lesões de pele encontradas no momento do diagnóstico: acima de cinco lesões classificado como MB e abaixo de cinco lesões, PB. Neste serviço, a classificação é feita pela composição do número de lesões de pele associando este indicador ao número de lesões de troncos nervosos. Estes diferentes modos de classificação, um utilizando apenas o número de lesões e outro o número de lesões mais o número de troncos nervosos acometidos, para o diagnóstico da hanseníase, podem alterar os resultados nos registros relativos à classificação operacional desta forma os dados epidemiológicos poderão estar divergentes influenciando no tratamento e no mecanismo das ações de controle.

Está posto nos resultados de todos os artigos selecionados uma taxa elevada de GIF como também do número de casos diagnosticados com a classificação operacional MB.

O número expressivo de pessoas afetadas pela hanseníase com incapacidades físicas também coloca a hanseníase incluída na lista das doenças negligenciadas, portanto requer mais atenção dos serviços e profissionais responsáveis por esta assistência, bem como um enfoque mais dirigido ao GIF no momento do diagnóstico dos casos novos de hanseníase. Considerar a utilização deste dado como um indicador significativo, como um preditor do diagnóstico tardio e um balizador da endemia, pode ser uma atitude a ser implementada.

Nos estudos do artigo E2 (Guerreiro et al, 2013) os autores qualificaram o tempo de demora para ser feito o diagnóstico como principal fator prognóstico e concluíram que é evidente o atraso no tempo do diagnóstico em pacientes que apresentaram incapacidade física. Possivelmente este fato também pode encobrir a realidade da prevalência e da própria situação da endemia, o que reforça a premissa desta revisão. Neste estudo é citada uma recomendação para que o GIF fosse utilizado como um indicador de diagnóstico tardio.

Ao investigarem os fatores associados ao atraso no diagnóstico através de entrevistas e revisão dos registros clínicos entre os anos de 2011 a 2015, os autores do artigo E3 (Gomez et al, 2018) também descreveram o registro do GIF como um indicador (parâmetro norteador) do diagnóstico tardio. Os autores deste artigo (E3) assinalam que ainda não existem estudos suficientes que mostrem a magnitude desta situação, esta questão está intimamente associada ao diagnóstico das incapacidades físicas no momento da primeira visita aos serviços de diagnóstico. Seria então um preditor (prenunciador)? Preditor que denuncia uma endemia oculta?

Os autores do artigo E4 (Oliveira et al, 2013) afirmam que a avaliação do grau de incapacidade física é um indicador dos programas para controle da hanseníase e que determina o diagnóstico seja precoce ou tardio, como também serve para monitorar o tratamento. O estudo mostra um dado em que o registro do exame do GIF no momento do diagnóstico foi realizado em 80,2\% do total dos casos novos selecionados pela amostra. Este dado pode apontar uma possível subnotificação e desenha o cenário dos registros nas unidades de referência observado no estudo E1, discutido acima, e que também citou a realização da avaliação neurológica em um número inferior ao da amostra pesquisada. Os autores do estudo E4 fazem uma ressalva sobre a deteç̧ão da deficiência física no momento do diagnóstico denuncia um prognóstico sombrio, além disto os autores propõem o exame do grau de incapacidade como fator preditor do diagnóstico tardio e suas complicações, como na proposição da presente revisão.

Nos resultados encontrados no estudo E5 (Alves et al, 2010), ao revisarem os prontuários dos casos novos do serviço, os autores observaram um predomínio de casos multibacilares e um elevado número de pacientes com incapacidade física no 
momento do diagnóstico, deste modo associam esta informação à pressuposição de que existe uma prevalência da hanseníase que não foi registrada, sugerindo uma possível endemicidade oculta. Neste trabalho a suposição de que existe uma endemia oculta devido ao excessivo registro de casos novos com incapacidades físicas está em consonância com a com as observações de outros autores analisados nesta revisão.

Os autores do artigo E6 (Silva et al, 2019) consideram o GIF um indicador indireto das ações que determinam um diagnóstico precoce da hanseníase. Citam a avaliação neurológica simplificada como o instrumento de eleição proposto pelo Ministério da Saúde/Brasil, mas destacam que é necessária uma qualificação das equipes profissionais para permitir um diagnóstico preciso e uma intervenção eficaz. Neste estudo, 18 prontuários foram excluídos devido à desacertos nos registros do GIF sinalizando as dificuldades na obtenção de dados fidedignos. De acordo com os autores deste artigo, estes dados delineiam um diagnóstico tardio. Do mesmo modo nos estudos E1, E2, E3, E4 e E5, os resultados assinalam um número elevado de GIF registrado no momento do diagnóstico, como também um número muito alto de casos multibacilares. O registro do GIF como indicador nos serviços de atendimento à hanseníase precisa ter um efeito confiável para permitir melhores resultados nos estudos epidemiológicos.

Corroborando com os autores do artigo E6, uma outra pesquisa com estudantes universitários de vários cursos da área da saúde, incluindo a medicina, realizada por Barros et al (2016), mostra nos resultados uma desinformação significativa em que a maioria dos entrevistados não identificavam quando havia um comprometimento neurológico na avaliação inicial das pessoas afetadas pela hanseníase. Neste trabalho está exposta outra questão significante que é o escasso interesse em desenvolver pesquisas nesta área, evidenciando a necessidade de um conhecimento mais aprofundado sobre a hanseníase na formação profissional e técnica, possibilitando atuações mais efetivas.

A incapacidade física presente no momento do diagnóstico do paciente com a classificação operacional MB determina que esta pessoa tem uma condição de transmissão prolongada pelo tempo de atraso no diagnóstico e é importante observar que este dado reflete o nível de capacidade dos serviços de detecção da doença segundo os autores do artigo E7 (Sarkar et al, 2012). Neste estudo o número de casos multibacilares diagnosticados coincidiu com uma maior quantidade de pessoas com incapacidades caracterizando que o atraso no diagnóstico aumentou a possibilidade destas pessoas desenvolverem deformidades.

Em todos os estudos, o GIF foi considerado um indicador essencial para a análise e supervisão da qualidade dos serviços e programas de assistência aos pacientes com hanseníase. Este estudo sugere uma reflexão sobre a utilidade do GIF como um indicador, um preditor, oferecendo informação para avaliar a eficácia das estratégias para o controle da hanseníase no intuito da implementação de medidas que provoquem ações para um diagnóstico precoce e consequente redução de danos.

\section{Conclusão}

Esta revisão evidencia o grau de incapacidade física como um importante indicador de diagnóstico tardio na medida em que os estudos analisados apresentam um percentual expressivo de incapacidades físicas associadas a um atraso no diagnóstico nos casos novos de hanseníase. A hanseníase carrega um estigma que se estende à vida pessoal e ocupacional das pessoas afetadas, muito agravado pela incapacidade física com repercussão psicoemocional e social significativas. Percebe-se que há necessidade de estratégias mais eficazes e propostas que despertem a promoção de ações mais concretas no âmbito das políticas públicas, na área acadêmica para a formação de técnicos e de profissionais nos centros universitários com vasto conhecimento da patologia, na capacitação de equipes assistenciais e na implementação de uma campanha mais robusta para informação da população. Estes são pontos fundamentais para quebrar esta cadeia da negligência às pessoas acometidas pela hanseníase. Neste sentido esta revisão acentua a necessidade de mais estudos, ações mais encadeadas, protocolos mais efetivos, equipes mais capacitadas, políticas de saúde mais dinâmicas e supervisionadas. 
Recomenda-se estudos futuros para investigar a associação entre a classificação operacional multibacilar e o grau de incapacidade física, bem como sobre a habilidade técnica dos profissionais de saúde na avaliação do grau de incapacidade física em pacientes com hanseníase.

\section{Referências}

Alves, C. J. M., Barreto, J. A., Fogagnolo, L., Contin, L. A. \& Nassif, P. W. (2010). Avaliação do grau de incapacidade dos pacientes com diagnóstico de hanseníase em serviço de dermatologia do estado de São Paulo. Revista da Sociedade Brasileira de Medicina Tropical,43(4), 460461. https://dx.doi.org/10.1590/S0037-86822010000400025.

Brandsma, W. J., \& Van Braekel, W. H. (2003). WHO disability grading: operational definitions. Leprosy Review. 74(4), 366 - 373.

Brasil. (2020). Boletim Epidemiológico: Secretaria de Vigilância em Saúde, Número Especial. Ministério da Saúde.

Brasil. (2020). Boletim Epidemiológico: Secretaria de Vigilância em Saúde, Ministério da Saúde. 51(28).

Brasil. (2016). Diretrizes para vigilância, atenção e eliminação da hanseníase como problema de saúde pública. Manual técnico-operacional.’ Ministério da Saúde.

Brasil. (2016). Diretrizes para vigilância, atenção e eliminação da Hanseníase como problema de saúde pública: Manual técnico-operacional. Ministério da Saúde.

Brasil. (2019). Secretaria de Vigilância em Saúde. coordenação-geral de Desenvolvimento da Epidemiologia em Serviço: Guia de Vigilância em Saúde. Volume único. Ministério da Saúde.

Brasil. (2019). Roteiro para uso do Sistema de Informação de Agravos de Notificação - Sinan NET para hanseníase. Manual para tabulação dos indicadores de hanseníase. Ministério da Saúde.

Brasil. (2020). Departamento de Doenças de Condições Crônicas e Infecções Sexualmente Transmissíveis. Estratégia Nacional para Enfrentamento da Hanseníase 2019-2022. Ministério da Saúde.

Gómez, L., Rivera, A., Vidal, Y., Bilbao, J., Kasang C. \& Parisi, S. et al. (2018). Factors associated with the delay of diagnosis of leprosy in north-eastern Colombia: a quantitative analysis. Trop Med Int Health. 23(2):193-198. 10.1111/tmi.13023.

Guerrero, M. I., Muvdi, S. \& León, C. I. (2013). Retraso en el diagnóstico de lepra como factor pronóstico de discapacidad en una cohorte de pacientes en Colombia, 2000-2010. Rev Panam Salud Publica. 2013;33(2):137-43.

Oliveira, D. T. D., Sherlock, J., Melo, E. V. D., Rollemberg, K. C. V., Paixao, T. R. S. D., Abuawad, Y. G. et al. (2013). Clinical variables associated with leprosy reactions and persistence of physical impairment. Revista da Sociedade Brasileira de Medicina Tropical, 46(5), 600-604.https://doi.org/10.1590/00378682-0100-2013.

Organização Pan-Americana da Saúde/Organização Mundial da Saúde (OPAS/OMS). (2017). Estratégia Global para Hanseníase 2016-2020.

Pessoa de Barros, P. M. F.; et al. (2016). The knowledge about leprosy by health undergraduate students at the public university in county Brazilian northeast. Hansen Int. 41(1-2): 14-24.

Peters, M. D. J., Godfrey, C., McInerney, P., Munn, Z., Tricco, A. C. \& Khalil, H. (2020). Revisões do escopo (versão 2020). In: Aromataris E, Munn Z (Editores). JBI Manual for Evidence Synthesis, 11. JBI. https://doi.org/10.46658/JBIMES-20-12.

Queirós, M. I., Ramos, J. A. N., Alencar, C. H. M., Monteiro, L. D., Sena, A.L. \& Barbosa, J. C. (2016). Clinical and epidemiological profile of leprosy patients attended at Ceará, 2007-2011. Anais Brasileiros de Dermatologia, 91(3), 311-317. https://dx.doi.org/10.1590/abd1806-4841.20164102.

Sarkar, J., Dasgupta, A., Dutt, D. (2012). Disability among new leprosy patients, an issue of concern: an institution based study in an endemic district for leprosy in the state of West Bengal, India. Indian J Dermatol Venereol Leprol. 78(3):328-34. 10.4103/0378-6323.95449.

Silva, J. S. R, Palmeira, I. P; Sá, A. M. M; Nogueira, L. M. V \& Ferreira, A. M. R. (2019). Variáveis clínicas associadas ao grau de incapacidade física na hanseníase. Revista Cuidarte, 10(1), e618. https://doi.org/10.15649/cuidarte.v10i1.618

Talhari, S. ; Penna, G. O; Gonçalves, H. S. ; Oliveira, M. L. W. (2015) Aspectos Gerais da Hanseníase, Agente Etiológico, Transmissão, Patogenia, Classificação, Manifestação Clínica, Diagnóstico. Hanseníase. Di Livros. (5) 194-172.

WHO. World Health Organization. (2017). The Operational Manual 2016 - Global Leprosy Estrategy, 2016-2020.

WHO. World Health Organization. (2009). Enhanced global strategy for further reduce the disease burden due to leprosy (plan period 2011-2015). Regional Office for South- East Asia, New Delhi.

WHO. World Health Organization. (2012). WHO Expert Committee on Leprosy. World Health Organization, Tech Rep Series.

WHO. World Health Organization. (2016). Global Leprosy Strategy: Accelerating towards a leprosy-free world.

WHO. World Health Organization. (2020). Global Leprosy Strategy: Overview 2021-2030.. Global Consultation NLP managers, partners and effected persons 26-30. 